\title{
Conditional versus Non-Conditional Incentives to Maximise Return of Participant Completed Questionnaires in Clinical Trials: A Cluster Randomised Study Within a Trial
}

Johanna Cook ( $\sim$ johanna.cook@phc.ox.ac.uk)

University of Oxford https://orcid.org/0000-0002-8750-8995

Jonathan A. Cook

University of Oxford

Emily Bongard

University of Oxford

Carl Heneghan

University of Oxford

Chris C. Butler

University of Oxford

\section{Research}

Keywords: Incentives, SWAT, Conditional Incentive, Non-Conditional Incentive, Participant Completed Questionnaire

Posted Date: June 25th, 2021

DOI: https://doi.org/10.21203/rs.3.rs-639815/v1

License: (c) (i) This work is licensed under a Creative Commons Attribution 4.0 International License. Read Full License 


\section{Abstract}

\section{Background}

High participant retention enhances the validity of clinical trials. A monetary incentive can increase retention, but it is not known if when it is provided matters. We aimed to determine whether there was a difference in the number follow-up trial questionnaires returned when a monetary incentive was given to participants at recruitment (non-conditional), compared to informing participants at recruitment that the incentive would be given only once a questionnaire had been returned (conditional).

\section{Method}

A sub-study within the Antivirals for influenza-Like Illness, An rCt of Clinical and Cost effectiveness in primary CarE (ALIC $\left.{ }^{4} E\right) \operatorname{Trial}^{2}$ Matched sites were randomised using computer generated random numbers, to either a non-conditional or conditional incentive. Analyses were conducted according to randomised group irrespective of compliance with two-sided $5 \%$ level statistical significance level. The main analysis was regression accounting for site pair, with additional weighted, paired and non-parametric sensitivity analyses. The total number of vouchers distributed, the cost of administration and postage was calculated.

\section{Results}

Of the 42 randomised sites (21 to each intervention) only 28 recruited at least one participant with only 10 practice pairs recruiting participants at both constituent sites. Raw diaries return proportions were 0.58 and 0.73 for non-conditional and conditional incentive groups. Regression analysis adjusted for cluster pair showed no significant difference in returns, $-0.09,(95 \% \mathrm{Cl},-0.29$, $0.10, p=0.336)$; when weighted there still no clear difference: $0.15(-0.02,0.31, p=0.068)$. There was no clear statistical evidence of a difference in time taken to return questionnaires, nor proportion of pages completed, by intervention group in the main analyses (all $p>0.05$ ). The conditional incentive was approximately $£ 23$ cheaper per diary returned based upon observed data.

\section{Conclusion}

There was no clear evidence of a difference in participant completed questionnaire returns according to the time at which an incentive is given, nor to completeness of the returned questionnaires, or the time to questionnaire return. There was substantial statistical uncertainty in findings, and some of the sensitivity analysis suggested that a meaningful benefit of a conditional incentive were plausible. The conditional approach cost less in cash terms.

\section{Background}

Completeness of follow up data is vital to trial validity. Despite this, most trials have important amounts of missing data (called 'loss to follow-up' or 'attrition'). [1] Higher levels of missing data increase the risk that the observed results are unrepresentative of the whole study group. [2] There are many reasons why data might be incomplete at the end of a trial; from trial sites not collecting all the data, to equipment failure, to data going missing or degrading during the time of the trial, and also participants not responding to questionnaires. The way that data is lost within a trial has differential effects on the potential bias assumed from that missing data with data "missing not at random" the most challenging to deal with. [3] When participants themselves do not respond to questionnaires this should be considered data that is "missing not at random" because there could be reasons for disproportionate levels of response from the different groups and, as a result, an increased likelihood of bias is assumed within the trial results.

There are many different methods that have been suggested or are used to improve retention rates in clinical trials, especially where the follow up is through participant completed questionnaires, but reports of effectiveness of many of these are anecdotal. [4] In a recent Cochrane review, Brueton et al, [5] examined the methods for improving lost to follow up that have been evaluated in randomised studies or quasai-randomised studies in health care. In the studies reviewed retention was listed as either a primary or secondary outcome. Of the trials identified, 14 evaluated incentives (monetary and non-monetary) that aimed to increase the response rate to participant completed postal questionnaires. They concluded that of all strategies investigated for the return of questionnaires, the addition of a monetary incentive increased responses the most. This confirms findings from the previous Cochrane Review by Edwards et al [6] that found that return of postal questionnaires more than doubled when a monetary 
incentive was provided. A conclusion of the Brueton et al [5] review was that more research was needed into methods for using incentives, and they expressed the need for more research into the optimal time monetary incentives should be provided, given the potential cost difference involved between provided an incentive initially for all, or just providing an incentive as a reward only for returned questionnaires. Trials currently being implemented in primary care vary in providing conditional $[7,8]$ or non-conditional $[9]$ incentives. Trial teams often debate which approach is optimal.

We therefore set out to examine the effect of adding in a monetary incentive of $£ 20$ as either conditional (given only once their questionnaire is returned), or non-conditional (given to all participants at the pint of recruitment) as a sub study nested within a large intentional randomised controlled trial, the Antivirals for influenza-Like Illness? An rCt of Clinical and Cost effectiveness in primary CarE (ALIC ${ }^{4} E$ ) Trial (ISRCTN:27908921). [10,11] Specifically, we aimed to determine the proportion of symptom diaries returned, the time to receipt of the diaries, the effect on pages completed, and cost differences between conditional and nonconditional approaches, including voucher costs and administration time.

\section{Method}

This was a cluster randomised matched-pair parallel-group Study Within A Trial (SWAT) $[12,13]$ within the ALIC ${ }^{4} E$ Trial. [10, 11] SWATs use the sample provided by the host trial to study the sub-question. As a result, they do not have their own sample size or power calculation. Our study proceeded according to a pre-specified protocol, that was approved by the research ethics committee as part of the main trial protocol, but which did not change during the course of the study.

$\mathrm{ALIC}^{4} \mathrm{E}$ is a European multi-national, multi-centre, phase IV, open-labelled, pragmatic, adaptive-platform, RCT. The trial aimed to determine whether adding antiviral treatment to best usual primary care is clinically and cost effective for patients with influenzalike illness. The trial was implemented in 21 networks across 15 countries in Europe. The follow up requirement within the ALIC ${ }^{4} \mathrm{E}$ Trial was a 14-day paper symptom diary (questionnaire) completed by the participants or their parent or legal guardian for the two weeks following recruitment into the study, then two further questionnaires completed via telephone call at days 14 and 28 . See Appendix 2 for $\mathrm{ALIC}^{4} \mathrm{E}$ inclusion and exclusion criteria and Appendix 3 for the full list of trial procedures.

We implemented the SWAT only in the UK networks that participated in the ALIC ${ }^{4} E$ Trial. Though collectively the recruiting networks achieved a return rate of the symptom diaries of over $90 \%$ in the first winter season of recruitment, within the UK sites the return rate was $59 \%$, meaning a loss to follow up rate of $31 \%$, far higher than the standardly accepted $20 \%$. [14] This was despite the same pre-planned follow up procedure for all networks. For the SWAT we indicated in the UK in participant information sheets that vouchers would be given to participants to thank them for their participation. We also indicated in the reminder texts for the participants in the conditional incentive group that once they returned their diary, they would receive their voucher. The recruiters were asked to complete receipts for all the vouchers dispensed so that the trial team could monitor compliance from the trial sites with voucher issue.

The main challenges in the design and implementation of this SWAT was ensuring that the individual participants received the vouchers at the appropriate time and that the randomisation arms had roughly equal levels of recruitment of participants into them. A cluster randomised design was used to enable the intervention to be applied consistently within sites for practical reasons. As there was concern about the relatively low number of clusters, and in order to try to manage anticipated differences between sites, which might impact upon recruitment, a matched pair cluster randomised design was used.

Table 1: Interventions and Comparators

\begin{tabular}{|l|l|}
\hline $\begin{array}{l}\text { Non- } \\
\text { Conditional } \\
\text { incentive arm }\end{array}$ & A $£ 20$ gift voucher given to study participants at the end of the recruitment visit (Baseline) \\
\hline $\begin{array}{l}\text { Conditional } \\
\text { incentive arm }\end{array}$ & $\begin{array}{l}\text { Participants informed that a } £ 20 \text { gift voucher would be given upon the return of the trial symptom } \\
\text { diary (Diary Return), and voucher then sent to those who returned a diary by the trial team }\end{array}$ \\
\hline
\end{tabular}


Table 2: Objectives and Outcome Measures

\begin{tabular}{|l|l|}
\hline $\begin{array}{l}\text { Primary Objective: } \\
\text { To assess the effect on study questionnaire return of giving a participant in an influenza } \\
\text { trial a } £ 20 \text { monetary incentive at the recruitment visit (non-conditional) or only once they } \\
\text { have returned their trial diary (conditional). }\end{array}$ & $\begin{array}{l}\text { Primary Outcome } \\
\text { Measure: } \\
\text { Proportion of } \\
\text { participants } \\
\text { returning a symptom } \\
\text { diary }\end{array}$ \\
\hline $\begin{array}{l}\text { Secondary Objective: } \\
\text { To assess the effect of an incentive on: }\end{array}$ & $\begin{array}{l}\text { Secondary Outcome } \\
\text { Measure: }\end{array}$ \\
$\begin{array}{l}\text { The time taken to return the participant diary } \\
\text { The proportion of the diary content completed }\end{array}$ & $\begin{array}{l}\text { 1. Time to diary } \\
\text { return } \\
\text { 2. The completeness } \\
\text { of the returned } \\
\text { diaries }\end{array}$ \\
\hline $\begin{array}{l}\text { Exploratory Objective: } \\
\text { To investigate the cost difference between conditional and non-conditional incentives }\end{array}$ & $\begin{array}{l}\text { Exploratory Outcome } \\
\text { Measures: } \\
\text { Costs incurred as a } \\
\text { result of the } \\
\text { incentives given }\end{array}$ \\
\hline
\end{tabular}

All outcomes were assessed after participants had completed their trial involvement. There were no changes to these outcomes after the SWAT commenced.

\section{Recruitment}

The ALIC ${ }^{4} E$ UK network included GP surgeries in Oxford, Southampton and Cardiff networks. The SWAT operated in 42 out of the $43 \mathrm{GP}$ Surgeries which recruited into the ALIC ${ }^{4} E$ Trial in the UK during the second and third seasons of the trial. One was excluded because of the non-availability of a further practice necessary for a matched pair. The site selected for exclusion was the last one recruited into the trial in Season 3. Recruitment of participants was during periods of heightened influenza activity, but all sites were randomised and trained prior to the participant recruitment beginning in season 2. Recruitment finished at the end of the third influenza season in 2018 and once the host trial had reached its recruitment target. Participants taking part in this SWAT had no additional requirements to those detailed above.

\section{Randomisation}

This SWAT used a matched pair cluster randomised parallel group design. The trial sites were the unit of randomisation. The sites were cluster randomised in order to keep the process as straight forward as possible for the recruiters. Randomisation was performed in two waves (before the start of seasons 2 and 3) using computer generated random numbers carried by one of the investigators (JAC). We paired the recruiting sites from Season 1 according to previous levels of recruitment and they were then randomised within these pairs prior the start of season 2. New sites starting during season 2 and 3 were randomised in matched pairs, but using the practice list size as the indicator of potential recruitment. Randomisation took place before the beginning of the second recruitment season. Sites continuing from season two carried on in their original randomised group. If there was to be an uneven number of sites and so an unpaired site, this site would continue on in the trial with the incentive given at a time more convenient for them, but they would not be included in the analysis of this SWAT. GP practices were not blinded to their allocation due to the necessity of them either distributing the incentives initially or not. Participants were unaware that there was a SWAT taking place as it was thought this might influence whether or not they returned their diary. See Appendix 1: Consort Diagram for details. 


\section{Statistical Analysis}

All statistical analyses were based upon the randomised groups irrespective of compliance with allocation at the cluster (site level). Overall and intervention group data were summarised using number of events and percentage for binary variables, mean and standard deviations (SDs) or median and interquartile ranges at site and participant level where appropriate. For all secondary outcomes, analyses were based also upon sites were at least one diary was returned. Due to the unanticipated small number of available pairs, the main analysis was carried out using regression with cluster option to account for site pair (a paired comparison was originally planned). A sensitivity analysis used the same model but weighted the observations according to number recruited or number of diaries returned depending upon the outcome. Sensitivity analyses were also used which included Mann-Whitney $U$ test on the site level data (ignoring the pairing) and two analyses on the subset of available paired data (paired $t$ test and a Wilcoxon sign rank test). 95\% confidence intervals (Cls) were calculated from the regression and the paired t-test analyses for the mean difference. Statistical significance was assessed at the 2-sided $5 \%$ significance level throughout. Recruitment data was available for all sites. Whether the Symptom diary was returned, and if it was, when it was received and how many pages were completed was available for all participants. There was no imputation of outcome data for sites where no-one was recruited, or where a participant did not return their Symptom diary

\section{Cost Analysis}

When assessing the cost of each intervention, a full economic analysis was not undertaken. We assessed the gross cost impact per diary in a simple cost analysis. When considering this there were different factors to take into account. The first and most important was the cost of the vouchers themselves, $£ 20$ per voucher dispensed. The indirect costs must also be taken into consideration, administration time and the cost of postage.

In the non-conditional arm it was the recruiter, most commonly a GP, who was giving out and completing the required forms and receipts for each voucher given. 5 minutes would be allowed for an administrative task such as this when considering NHS service support cost reimbursements. At this time a GPs time was reimbursed at $£ 80$ per hour according to the NHS research reimbursement figures.

In the conditional arm the amount of time required for the administration was a similar 5 minutes, but this task would be completed by an administrator whose time was charged at $£ 21$ per hour. In addition to this there was the postal cost of sending the voucher to the participants for which the cost of a second class letter was $£ 0.56$.

To assess the impact of the direct and indirect costs we took into account the number of times each item or activity was required, according to the number of vouchers given and so calculated a total cost per arm and then a total cost per questionnaire returned. No formal analysis of the results were carried out and the cost per diary returned for each group was calculated.

\section{Results}

Table 3

Baseline Characteristics of sites

\begin{tabular}{|lll|}
\hline & Non-Conditional Incentive Arm ( $\mathbf{n = 2 1 )}$ & Conditional Incentive Arm ( $\mathbf{n = 2 1 )}$ \\
\hline $\begin{array}{l}\text { Previous Recruitment }- \text { mean (range) } \\
\text { (for those recruited in Season 1) }\end{array}$ & $5(1-19), n=9$ & $4(1-14), n=9$ \\
\hline Practice List Size - median (IQR) & $9674(7900,15408)$ & $12055(7050,15600)$ \\
\hline
\end{tabular}




\begin{tabular}{|lll|}
\hline & $\begin{array}{l}\text { Non-Conditional Incentive Arm }(\mathbf{n = 2 2 0} \text { unless } \\
\text { otherwise stated })\end{array}$ & $\begin{array}{l}\text { Conditional Incentive Arm }(\mathbf{n}=\mathbf{1 2 5} \text { unless } \\
\text { otherwise stated })\end{array}$ \\
\hline $\begin{array}{l}\text { Duration of Illness (days) }- \\
\text { median (IQR) }\end{array}$ & $2(2,3)$ & $3(2,3)$ \\
\hline $\begin{array}{l}\text { Score of clinical Severity }- \\
\text { median (IQR) }\end{array}$ & $2(1,2)$ & $2(2,2)$ \\
\hline Age - median (IQR) & $26(12,46)$ & $36(23,53)$ \\
\hline Temperature - median (IQR) & $37.1(36.6,37.6)$ & $37.6(37.0,38.2), \mathrm{n}=124$ \\
\hline Female - $\mathrm{n}(\%)$ & $135(61)$ & $73(58), \mathrm{n}=124$ \\
\hline
\end{tabular}

The baseline characteristics of previous recruitment was relatively well balanced though practice size was not (Table 3). The baseline characteristics of the participants within the SWAT were well balanced (Table 4). The baseline participant level data was reasonably well balanced between intervention groups.

Of the 42 sites involved in the study, only twenty-eight (67\%) sites recruited at least one participant. Only 10 of the 21 (48\%) site pairs had at least participant recruited in both sites within the pair. The median (IQR) numbers recruited across all sites was 2 $(0,10)$, and by conditional and non-conditional arms were $3(0,10)$ and $3(0,10)$ respectively.

\section{Compliance}

All participants in the conditional incentive group received their vouchers. Within non-conditional group there were 5 participants who did not receive the vouchers as they should have done. This was due to site non-compliance and non-response to the trial team who were trying to restock their vouchers. The site were re-trained and the issue did not occur again.

Table 5

Overall Recruitment and outcomes by Intervention Group at the participant level

\begin{tabular}{|lllll|}
\hline & $\begin{array}{l}\text { Participants recruited across } \\
\text { sites }\end{array}$ & $\begin{array}{l}\text { Diaries received } \\
\text { across sites }-\mathbf{n} \\
(\%)\end{array}$ & $\begin{array}{l}\text { Days to diary } \\
\text { return - } \\
\text { median (IQR) }\end{array}$ & $\begin{array}{l}\text { Number of pages } \\
\text { completed - } \\
\text { median (IQR) }\end{array}$ \\
\hline $\begin{array}{l}\text { Non-Conditional } \\
\text { Incentive }\end{array}$ & 220 & $127(58)$ & $23(19,27)$ & $35(35,35)$ \\
\hline Conditional Incentive & 125 & $91(73)$ & $20(18,25)$ & $35(35,35)$ \\
\hline Total & 345 & $218(63)$ & $21(18,27)$ & $35(35,35)$ \\
\hline
\end{tabular}

Return of Diaries

Twenty six of the twenty eight (93\%) sites that recruited at least one participant returned one or more diaries; the proportion returned varied from 0 to 1.0 within both intervention groups reflecting the small number recruited per site. Median and IQR site proportions returned were $0.66(0.57,1.00)$ and $0.71(0.50,0.80)$ for the non-conditional and conditional incentive groups respectively. The raw proportion returned irrespective of site was 0.58 and 0.73 for non-conditional and conditional incentive groups. Corresponding values for the subset (10 pairs) where both of the constituent sites paired at randomised recruited one or more participant were 0.56 and 0.73 . The median and IQR for the aforementioned subset of 10 pairs was $0.64(0.57,1.00)$ and $0.71(0.50,0.92)$ for non-conditional and conditional incentive group respectively.

Regression analysis adjusted for cluster pair was a difference between interventions of $-0.09,95 \% \mathrm{Cl}(-0.29,0.10), \mathrm{p}=0.336$. The sensitivity analysis which also weighted by the number of individuals recruited at each site produced a substantial different raw effect though it was still not statistically significant: $0.15,95 \% \mathrm{Cl}(-0.02,0.31), \mathrm{p}=0.068$. Non-parametric test Wilcoxon signed rank test gave a similar finding of no clear statistical evidence of a difference $(p=0.760)$. The other sensitivity analyses which ignored 
the pairs and analysed all sites where one or more participant was recruited also gave similar findings with Mann-Whitney $p=$ 0.695 , and unweighted paired t test had a mean difference of $-0.04,95 \% \mathrm{Cl}(-0.28,0.19), \mathrm{p}=0.701$.

Time to Diary Return

The individual time to return ranged from 10 to 64 days across both intervention groups. Median and IQR of the average times to return by intervention group at site level were $23(20,26)$ days and $21(19,25)$ days for non-conditional and conditional incentive group respectively. Corresponding values for the subset (9 pairs) where both constituent sites had at least one diary returned were $24(23,25)$ days and $21(19,24)$ days for non-conditional and conditional incentive group respectively.

Regression analysis adjusted for cluster pair was a mean difference between interventions of $-0.9,95 \% \mathrm{Cl}(-5.7,3.8)$ days, $\mathrm{p}=$ 0.685. The sensitivity analysis which also weighted by the number of individuals recruited at each site produced a substantial different raw effect which was statistically significant: $-3.1,95 \% \mathrm{Cl}(-5.0,-1.1), \mathrm{p}=0.004$. Non-parametric test Wilcoxon signed rank test gave a similar finding $(p=0.214)$. The other sensitivity analyses which ignored the pairs and analysed all sites where one or more participant was recruited also gave similar findings with Mann-Whitney $p=0.354$, and unweighted paired $t$ test had a mean difference of $-0.8,95 \% \mathrm{Cl}(-7.0,5.4)$ days; $\mathrm{p}=0.768$.

Number of Pages Completed

The average number of pages completed per diary returned varied from 7 to 36 pages across both intervention groups. Median and IQR average pages completed by intervention group at site level were $35(34,35)$ pages and $35(35,35)$ pages for nonconditional and conditional incentive group respectively. Corresponding values for the subset (9 pairs) where both constituent sites had at least one diary returned were $35(34,35)$ pages and $35(35,35)$ pages for non-conditional and conditional incentive group respectively. When returned, the diary was generally fully completed or almost fully complete; only 7 (3\%) across all participants failed to return at least 30 pages when diary was returned. By intervention group this was $4(3 \%)$ and $3(3 \%)$ respectively for nonconditional and conditional intervention groups.

Regression analysis adjusted for cluster pair was a mean difference between interventions of $0.5,95 \% \mathrm{Cl}(-1.2,2.3)$ pages, $\mathrm{p}=$ 0.519 . The sensitivity analysis which also weighted by the number of diaries returned at each site had a similar result: $0.3,95 \% \mathrm{Cl}$ $(-0.7,1.2), p=0.552$. Non-parametric test Wilcoxon signed rank test gave a similar finding $(p=0.152)$. The other sensitivity analyses which ignored the pairs and analysed all sites where one or more participant was recruited also gave similar findings with Mann-Whitney $p=0.176$, and unweighted paired t test had a mean difference of $0.20,95 \% \mathrm{Cl}(-0.7,1.1)$ pages; $p=0.612$.

\section{Cost Analysis}

In the non-conditional incentive arm there were 220 participants recruited, of these 215 received a $£ 20$ voucher despite only receiving 127 diaries back. Therefore the total cost for the vouchers was $£ 4,300$ and for each questionnaire actually returned the cost was $£ 33.85$. In the conditional incentive arm, because vouchers were only sent out once a diary was received, the total cost for the vouchers was $£ 1,820$ and the cost for each diary received is $£ 20.00$.

For the indirect costs in the non-conditional arm with 215 vouchers distributed the total cost for the administration time was $£ 1,433$ and $£ 11$ per diary received. For the conditional arm the total cost of the administration was roughly $£ 159$ and $£ 1.75$ per diary received. The total additional cost of the second class letter was $£ 50.96$ making a total of roughly $£ 2.31$ per diary received when considering administration and postage.

Therefore, the total cost for the non-conditional incentive arm was $£ 5,733$ and per diary received was estimated to be $£ 45$. For the conditional incentive arm the total was estimated to be around $£ 2,029$ with a per diary cost of $£ 22$ a total difference of $£ 23$ in favour of the conditional arm.

\section{Discussion}

Impact on current literature 
This was the first study to compare the effect of conditional versus non-conditional monetary incentives on return and completion of a participant completed questionnaire in a RCT. There was no significant difference in the proportion of questionnaires returned between the times at which the incentive is given is in line with the current, limited, research. However, the raw difference of a $15 \%$ improvement ( $17 \%$ for the subset of site pairs where both recruited one or more) diaries returned in favour of the conditional incentive arm would be a meaningful one to trialists if genuine. This along with the confidence intervals for the site level analyses being relatively wide suggest that the analysis lack precision and that the possibility of a genuine and operationally important difference (to trialists) cannot be ruled out. Sensitivity analyses which weighted by the number of observations which contributed to the site summary suggest that such an effect is quite possible. This needs exploring in further studies and incorporating this data ultimately within an updated systematic review of incentive use for retention. Additionally the cost difference between the two approaches suggests that a conditional incentive is more economical.

\section{Strengths}

This SWAT was implemented into the ALIC ${ }^{4} E$ UK networks with very little to no impact on the trial, the study team and the recruiting sites and with none on the participants themselves. Compliance was very high which to a degree probably reflected the use of a cluster randomised design which made this much easier to administer, particularly part way through the study.

\section{Limitations}

The sample size was relatively small, with only 345 participants recruited during the SWAT, and therefore the study risks Type II error. This is a general design challenge for of SWATs, where no power calculation is done for the sub-study itself and the recruitment is dependent upon the host trial.

Using the GP Surgeries as the unit of randomisation was best for the practical implementation of the study, especially with consideration to the host trial and not wanting to compromise the main aims of that investigation. It was also an attractive method to use to overcome the known risk of imbalance due to differences between sites, but this in turn, created its own difficulty. The way that the pairing of these sites worked out meant that there were many pairs with a site or both sites having not recruited. This led to only being able to use results from 10 of the 21 pairs recruited within the initial planned analysis. With the benefit of hindsight, the matched pair design was not the optimal choice due to the substantial number of sites who failed to recruit any participants and therefore did not contribute data to the analysis. In retrospect, this design in a primary care setting, where there is normally a relatively high number of sites that do not recruit any participants, was not well suited to the context. The limitations of the design in another context (community trials) has been noted. [15] It could work within secondary care, where most sites do recruit at least one or two participants. The paired design was chosen given the limited number of sites available but a potentially different design could have been to do either simple randomisation of all included sites or a stratified randomisation approach based again upon recruitment level; either may well have worked better. It is interesting to note that previous recruitment does not seem to have been a very strong predictor of future recruitment at least at the level of being useful for matching.

Analysing data from matched paired cluster trials is surprisingly difficult as Donner et al show. [16] To simplify the statistical analysis, and without likely loss of statistical precision given the relatively small number of clusters and observations within clusters, all analyses were conducted at the cluster (primary care surgery) level. The sensitivity analyses using regression with cluster robust variance and weighting for the number of contributing observations may have been the most appropriate analysis in this content given the difficulties with the matching and low recruitment per site. An participant level analysis would have been another options which was not utilised here.

The level of compliance with voucher issue was high within this study, though not $100 \%$. There were five participants within the non-conditional group that did not receive their voucher, none of these participants returned their diary. This lack of compliance could therefore have impacted upon the result. However, the analysis was carried out with these five participants included on an intention to treat as is generally recommended. Given the small number seems unlikely to have affected the findings. No imputation for missing data was made which was a consequence of the design in terms of some site not recruiting anyone. Had more sites been involved perhaps grouping practice may have helped avoid this though possibly at the risk of greater imbalance between intervention groups. 


\section{Conclusion}

There was no clear evidence of a difference in retention rate according to when a participant is given a monetary incentive for participant completed questionnaire return; however our study may have lacked precision given the small number of clusters and that a substantial proportion did not recruit any participants. Additionally, there was no clear statistical evidence of a difference in the completion of the diary or questionnaire and the speed with which they were returned this to the trial team. However, the analysis lacked precision, and sensitivity analyses which accounted for imbalances between sites suggested genuine effect are plausible. The possibility of a genuine and operationally important difference (to triallists) cannot be ruled out. Furthermore there was, as expected, a large difference between the gross costs of giving a monetary voucher as a non-conditional incentive as opposed to a conditional incentive in simple monetary terms. It cost approximately $£ 23$ more for every diary received to give the vouchers as a non-conditional incentive. It may be cost effective to give the incentives at the point at which a diary or questionnaire is received by the trial staff than when the participant is recruited. Further studies are needed to explore this aspect of clinical trial conduct.

\section{List Of Abbreviations}

\begin{tabular}{|l|l|}
\hline${ }_{\text {ALIC }}^{4} \mathrm{E}$ & Antivirals for influenza-Like Illness? An rCt of Clinical and Cost effectiveness in primary CarE \\
\hline RCT & Randomised Controlled Trial \\
\hline SWAT & Study Within A Trial \\
\hline
\end{tabular}

\section{Declarations}

\section{Ethics approval and consent to participate}

The trial, as well as this SWAT, was granted research ethics committee (REC) approval by the NRES Committee South Central (Oxford B). All participants provided written informed consent before participation in the main trial. The study was conducted according to the principles of the Declaration of Helsinki and in accordance with other relevant national guidelines, regulations, and acts.

\section{Consent for publication}

Not required.

\section{Availability of data and material}

The datasets used and/or analysed during the current study are available from the corresponding author on reasonable request.

\section{Competing interests}

The authors have no competing interests

\section{Funding}

This work was supported by the European Commission FP7 Programme grant no HEALTH-F3-2013-602525. The funding body played no part in the design of the study and collection, analysis, and interpretation of data and in writing the manuscript.

\section{Authors' contributions}


$\mathrm{JC}$ came up with the initial idea for the study, developed the study protocol, and set-up the systems for implementation, carried out data cleaning, over saw the analysis and wrote up this dissertation. CB and $\mathrm{CH}$ helped develop the protocol. EB helped implement the study, with help from the ALIC ${ }^{4} E$ Trial team, particularly the Oxford, Southampton and Cardiff co-ordinating centres. Statistical advice generation random allocations and carrying out the statistical analyses was undertaken by JAC. All authors have commented on this paper.

\section{Acknowledgements}

In addition to the authors, we would like to acknowledge the contribution of the other members of the 3 ALIC ${ }^{4} E$ co-ordinating centres involved in this SWAT: Department of Primary Care Health Sciences and Primary Care Clinical Trials Unit, University of Oxford, Ely Bridge Surgery, Cardiff, Primary Care and Population Science, University of Southampton, Southampton; in particular Sonya Beecher, Mel Davies, Jane Barnett, respectively, for their administrative and trial management support.

\section{References}

1. Dumville JC. Reporting Attrition in Randomised Controlled Trials. BMJ. 2006;332:969.

2. Dettori J. Loss to Follow-up. Evidence Based Spinal Care Journal. 2011;2(1):7-10.

3. Altman DG. Missing Data. BMJ. 2007;334(7590):424.

4. Norvell DC. Enhancing Clinical Study Retention Rates to Avoid Follow-up Bias: How Do We Keep Our Study Participants from "The Land of the Lost"? Global Spine Journal, 2016, 519-521.

5. Brueton VC. Strategies to improve retention in randomised trials. Cochrane Database Systematic Reviews, $2013,(12): 1-126$.

6. Edwards PJ. Methods to increase response to postal and electronic questionnaires. Cochrane Database Systematic Reviews, 2009, Jul 8;(3).

7. Cook J. Oral corticosteroid use for clinical and cost-effective symptom relief of sore throat: study protocol for a randomized controlled trial. Trials. 2014;15:365.

8. Downing HE. Can oral corticosteroids reduce the severity or duration of an acute cough, and the associated National Health Service and societal costs, in adults presenting to primary care? Study protocol for a randomised controlled trial. Trials. 2015;16:78.

9. Spanou C. Preventing disease through opportunistic, rapid engagement by primary care teams using behaviour change counselling (PRE-EMPT): protocol for a general practice-based cluster randomised trial. BMC Family Practice. 2010;11:69.

10. Buter C. Oseltamivir plus usual care versus usual care for influenza-like illness in primary care: an open-label, pragmatic, randomised controlled trial. The Lancet, Volume 395, ISSUE 10217, P42-52, January 04, 2020 doi:https://doi.org/10.1016/S0140-6736(19)32982-4.

11. Bongard E. Antivirals for influenza-Like Illness? A randomised Controlled trial of Clinical and Cost effectiveness in primary CarE (ALIC4 E): the ALIC ${ }^{4}$ E protocol. BMJ Open. 2018. doi:10.1136/bmjopen-2017-021032.

12. MRC. The Northern Ireland Hub for Trials Methodology Research. Retrieved from The Northern Ireland Hub for Trials Methodology Research: https://www.qub.ac.uk/sites/TheNorthernIrelandNetworkforTrialsMethodologyResearch/SWATSWARInformation/ Accessed 22 Jul 2019.

13. Cook JSWAT 90: Does the time at which a participant incentive is given affect the retention rate? In: The MRC SWAT Store, MRC Methodology Hub 2019, https://www.qub.ac.uk/sites/TheNorthernIrelandNetworkforTrialsMethodologyResearch/FileStore/Filetoupload,902359,en.pdf Accessed 22 Jul 2019.

14. CEBM.. Levels of Evidence, Retrieved from Centre for Evidence Based Medicine: Oxford CEBM. http://www.cebm.net/levels_of_evidence.asp Accessed 26 Mar 2018.

15. Klar N. The Merits of Matching in Community Intervention Trials: a cautionary tale. Stat Med. 2015;16(15):1753-64. 
16. Donner A. Confidence Interval Construction for Effect Measures Arising from Cluster Randomisation Trials. Journal of Clinical Epidimiology. 1993;46:123-31.

\section{Supplementary Files}

This is a list of supplementary files associated with this preprint. Click to download.

- Appendix.docx

- CONSORTExtensionforClusterTrials2012Checklist.docx 